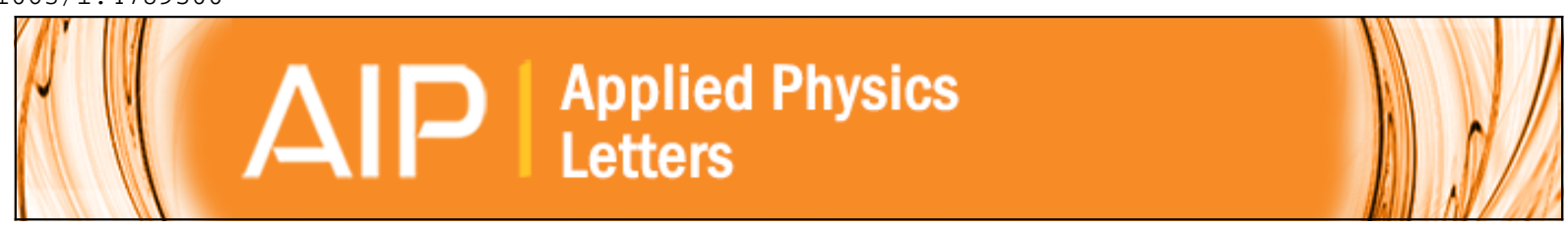

\title{
Stress reconfigurable tunable magnetoelectric resonators as magnetic sensors
}

Jillian Kiser, Peter Finkel, Junqi Gao, Christophe Dolabdjian, Jiefang Li, and D. Viehland

Citation: Applied Physics Letters 102, 042909 (2013); doi: 10.1063/1.4789500

View online: http://dx.doi.org/10.1063/1.4789500

View Table of Contents: http://scitation.aip.org/content/aip/journal/apl/102/4?ver=pdfcov

Published by the AIP Publishing

$\underset{\substack{\text { Aubloning } \\ \text { Alp }}}{A}$ Re-register for Table of Content Alerts 


\title{
Stress reconfigurable tunable magnetoelectric resonators as magnetic sensors
}

\author{
Jillian Kiser, ${ }^{1}$ Peter Finkel, ${ }^{1, a)}$ Junqi Gao, ${ }^{2}$ Christophe Dolabdjian, ${ }^{3}$ Jiefang $\mathrm{Li},{ }^{2}$ \\ and D. Viehland ${ }^{2}$ \\ ${ }^{1}$ Naval Undersea Warfare Center, Newport, Rhode Island 02841, USA \\ ${ }^{2}$ Materials Science and Engineering, Virginia Tech, Blacksburg, Virginia 24061, USA \\ ${ }^{3}$ Université de Caen Basse-Normandie, ENSICAEN and CNRS, UMR6072 GREYC, F-14032 Caen, France
}

(Received 21 November 2012; accepted 14 January 2013; published online 31 January 2013)

\begin{abstract}
We report a magnetoelastic effect in doubly clamped ferromagnetic magnetostrictive Metglas resonators with electrically and magnetically reconfigurable frequency response. The field-induced resonance frequency shift is due to magnetostrictive strain, which is shown to have a strong dependence on uniaxial stress. Here, we demonstrate that this magnetic field induced behavior can be used as the basis for a simple, tunable, magnetoelectric magnetic field sensor. The effect of tension on the field dependent magnetostrictive constant and the sensor sensitivity is examined, and the equivalent magnetic noise floor of such a sensor is estimated. (c) 2013 American Institute of Physics. [http://dx.doi.org/10.1063/1.4789500]
\end{abstract}

Multiferroic magnetoelectric (ME) composites have been the subject of much research for various applications, including multifunction transducers and sensors. ${ }^{1-10}$ It has been seen that the strongest ME coupling is often achieved not with single phase materials, but using strain-mediated ME composite systems of magnetostrictive and piezoelectric layers, ${ }^{11}$ which have been designed to generate giant $\mathrm{ME}$ coefficients. ${ }^{6-10}$ Under magnetic field, strain generated by a magnetostrictive layer is transmitted to a piezoelectric layer resulting in a change in electric polarization. Accordingly, a magnetic signal is converted into an induced voltage that can be utilized to sense and measure magnetic fields. Many devices have been designed to utilize giant ME coupling coefficients for low frequency passive magnetometry, establishing equivalent magnetic noise floors as low as $40 \mathrm{pT} / \sqrt{ } \mathrm{Hz}$ at $f=1 \mathrm{~Hz} .{ }^{12}$ Magnetic field sensors based on the ME effect are particularly promising due to relatively high sensitivity compared with traditional magnetometers (Fluxgate, giant magnetoresistance (GMR), and giant magnetoinductance (GMI)), as well as benefiting from low power requirements as a result of their passive nature. ${ }^{10}$ The sensitivity of this type of sensor relies on the efficiency of the ME coupling, as determined by the magnetoelectric coefficient $\alpha_{M E}$. This coefficient represents the coupling between the piezoelectric and magnetoelastic components and is defined as the change in electric field $E$ as a function of change in magnetic field $H$, i.e., $\Delta E / \Delta H$. The effective value of $\alpha_{M E}$ is a function of the piezomagnetic coefficient $d_{m}=\partial \lambda / \partial H$, where $\lambda$ is the magnetostriction constant. Maximum sensitivity with minimal applied $H_{d c}$ can be achieved by maximizing $d_{m}$ at low magnetic field bias $H_{d c}$. For this characteristic, Metglas (FeCoSiB) has been chosen as the magnetostrictive component in several reports ${ }^{9,12,13}$ and also in this study.

One way to increase resolution and reduce the impact of magnetic and environmental noise is to operate at the elec-

\footnotetext{
${ }^{\text {a) }}$ Author to whom correspondence should be addressed. Electronic mail: peter.finkel@navy.mil.
}

tromechanical resonance (EMR), thus enhancing $\alpha_{M E}{ }^{7,14}$ Investigations have shown that $\alpha_{M E}$ may be enhanced by up to two orders of magnitude at EMR. ${ }^{15}$ However, the advantages of driving the sensor at resonance are only available over a narrow bandwidth. Broadband operation at resonance can be achieved by tuning the resonance frequency, either by changing the mass or stiffness of a free cantilever structure ${ }^{14}$ or by adjusting the mechanical stress of a bridge-like structure. ${ }^{15-17}$ For example, Finkel et al. achieved a full octave of tuning capability by utilizing a uni-axial stress tuning approach in double-clamped ME structures. ${ }^{18}$ As will be seen, the magnetic field frequency dependence of tunable resonators can be used for near-DC magnetic field sensing.

One advantage of stress-reconfigurable resonance tuning is the access to higher resonance frequencies, which enables superior $1 / f$ noise rejection. However, the benefit of noise rejection at higher resonance frequencies is offset by the reduction in $\alpha_{M E}$ and a resultant loss of sensor sensitivity at higher stress levels. ${ }^{18}$ Therefore, high resonance frequencies are more efficiently achieved by decreasing sensor dimensions, rather than applying high levels of stress.

In this letter, we present magnetoelastic properties of doubly clamped Metglas resonators and the field dependence of the flexural resonance frequency as a function of uni-axial stress. Potential noise sources and the sensor sensitivity are also investigated. A strip of Metglas $\left(\mathrm{Fe}_{74.4} \mathrm{Co}_{21.6} \mathrm{Si}_{0.5} \mathrm{~B}_{3.3} \mathrm{Mn}_{0.1} \mathrm{C}_{0.1}\right)$ $(50 \times 5 \times 0.025 \mathrm{~mm})$ is clamped at each end and is excited using a PZT element in a custom designed sample loading fixture (Figure 1(a)). ${ }^{18}$ This sample holder is positioned in a Helmholtz coil, which provides a magnetic field bias $\left(H_{d c}\right)$ up to 120 Oe. The magnetic field is oriented parallel to the long axis of the sample, which provides the greatest magnetostrictive response. The transverse vibrational mode of the sample is measured with a laser Doppler vibrometer (LDV) (Polytec OFV-5000).

To evaluate the effect of tension on the sample, a bias voltage on the PZT element was varied, such that the resonance frequency at $H_{d c}=0$ varied from 550 to $850 \mathrm{~Hz}$. The 
resonance frequency is tracked in real time using a lock-in amplifier (LIA) (SR850) and a voltage controlled oscillator (VCO) (HP3314a) feedback loop, as shown in Figure 1(b). The LIA calculates the quadrature component of the sample motion, using the driving signal as a reference. This produces a positive DC output at driving frequencies lower than the sample resonance and a negative output when the driving frequency is greater than the resonance frequency. The DC output is fed into the VCO, which produces the driving signal for the PZT element in the sample holder. This negative feedback loop stabilizes when the quadrature component is zero, which occurs at the sample's resonance frequency.

For each level of tension, $H_{d c}$ is manually swept from 0 to \pm 120 Oe and back over a period of approximately $120 \mathrm{~s}$. The resonance frequency is tracked using the LIA-VCO feedback loop described above, and data are recorded at $512 \mathrm{~Hz}$ using a data acquisition board (NI BNC-2010). The resonance frequency values are recorded simultaneously with $H_{d c}$, as measured by a Lakeshore 450 Gaussmeter.

The magnetostriction constant, $\lambda$, can be calculated by modeling the strip of Metglas as a one dimensional string, resonating in the first fundamental mode. Though this simplification would not account for an anisotropic internal stress state or non-ideal boundary conditions, it will serve as a first order approximation of resonance. In the case of a tensioned

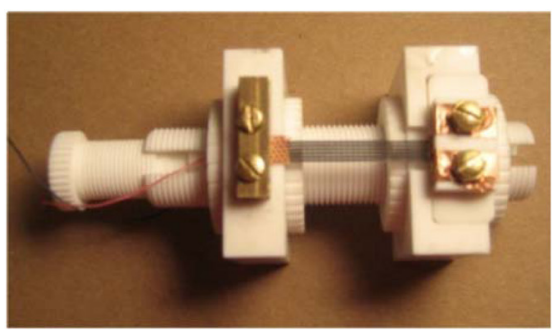

(a)

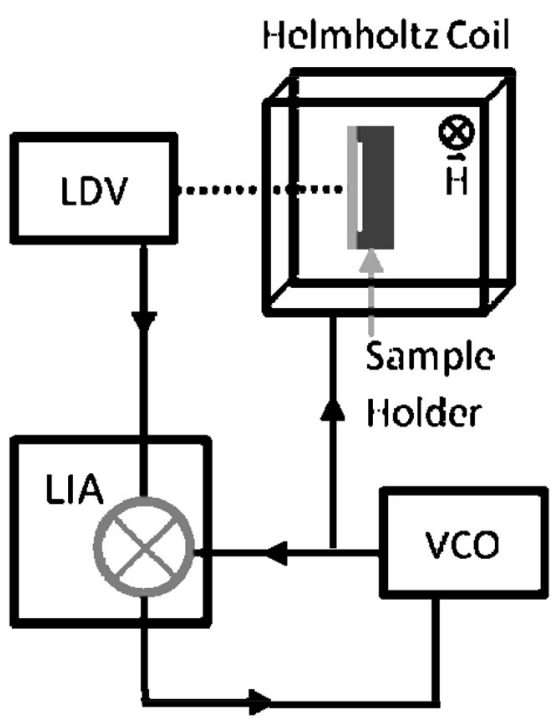

(b)

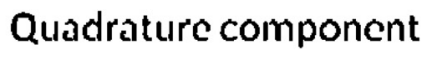

FIG. 1. (a) Sample holder (adapted from Ref. 19), with Metglas sample installed. Metglas is clamped at each end and excited by an internal PZT stack placed inside the fixture (not shown). The tension can be manually adjusted with the screw on the left. (b) Schematic of experimental set-up and feedback loop. The dotted line indicates the laser beam from the LDV (see text). string, the resonance frequency $f_{0}$ is given by $(\sigma / \rho)^{1 / 2}(2 L)^{-1}$, where $\sigma$ is the tension, $\rho$ is the density, and $L$ is the length of the Metglas strip. Application of DC magnetic field results in a change in $f_{0}$ due to tension changes, which can be written as

$$
f=\frac{1}{2 L} \sqrt{\frac{\sigma-\lambda(H, \sigma) E}{\rho}},
$$

where $E$ is the elastic modulus and $\lambda$ is the magnetostriction constant, which is a function of both tension and magnetic field. Rearranging terms to solve for $\lambda$, we find

$$
\lambda=\left[1-\left(\frac{\Delta f}{f_{0}}\right)^{2}\right] \frac{\sigma}{E},
$$

where tension is equal to $4 L^{2} f_{0}^{2} \rho, \Delta f$ is the resonance frequency shift due to magnetic field bias, and $f_{0}$ is the resonance frequency with zero magnetic field bias. Additionally, this "taut string" model can be used to predict the maximum shift in resonance frequency $\Delta f_{\text {sat }}$, due to magnetic field saturation, which can be calculated as

$$
f_{s a t}-f_{0}=\Delta f_{s a t}=\frac{1}{2 L \sqrt{\rho}}\left(\sqrt{\sigma}-\sqrt{\sigma-\lambda_{s a t} E}\right) .
$$

Figure 2 shows the change in resonance frequency of a doubly clamped Metglas sample as a function of $H_{d c}$ at four levels of tension. Using these data, $\lambda$ can be examined as a function of either $\sigma$ or $H$. In Figure 3(a), it is seen that $\lambda$ increases with magnetic field until Metglas reaches magnetic saturation at approximately $20 \mathrm{Oe}$. The piezomagnetic coefficient $d_{m}(\partial \lambda / \partial H)$ can also be calculated as a function of magnetic field. As seen in Figure 3(a), $d_{m}$ results in maximum piezomagnetic coupling at $H_{d c}=7 \mathrm{Oe}$.

The effect of tension on $\lambda$ can be examined by focusing on the resonance frequency shift resulting from a saturating magnetic field. As seen in Figure $3(\mathrm{~b}), \lambda_{\text {sat }}$ decreases with increasing tension, which reflects a corresponding decrease in $\Delta f$. This suggests that, for maximum sensitivity of the sensor, the tension value should be kept to a minimum. By using an approximation $\lambda_{\text {sat }} \sim \sigma^{-1}, \lambda_{\text {sat }}$ can be extrapolated to the value of $21 \mathrm{ppm}$ at $\sigma=0$. To validate the extrapolated value,

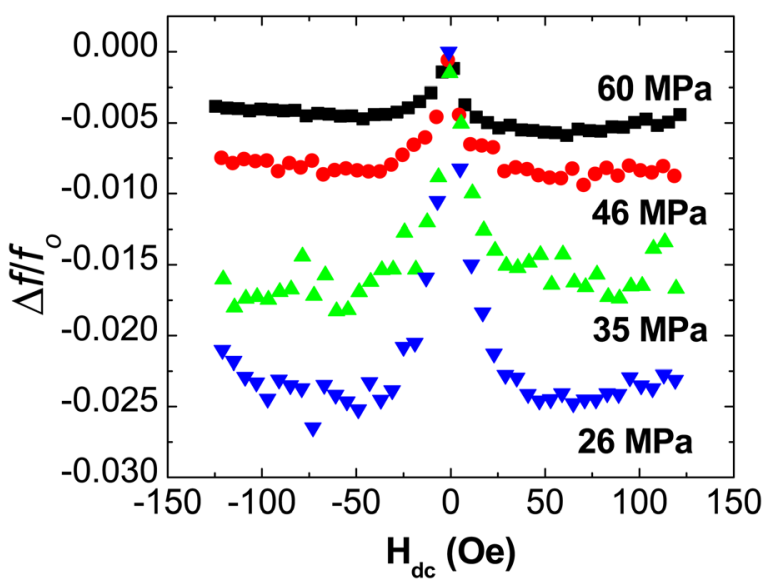

FIG. 2. Frequency shift vs. $H_{d c}$ as a function of uni-axial stress $\sigma$. 

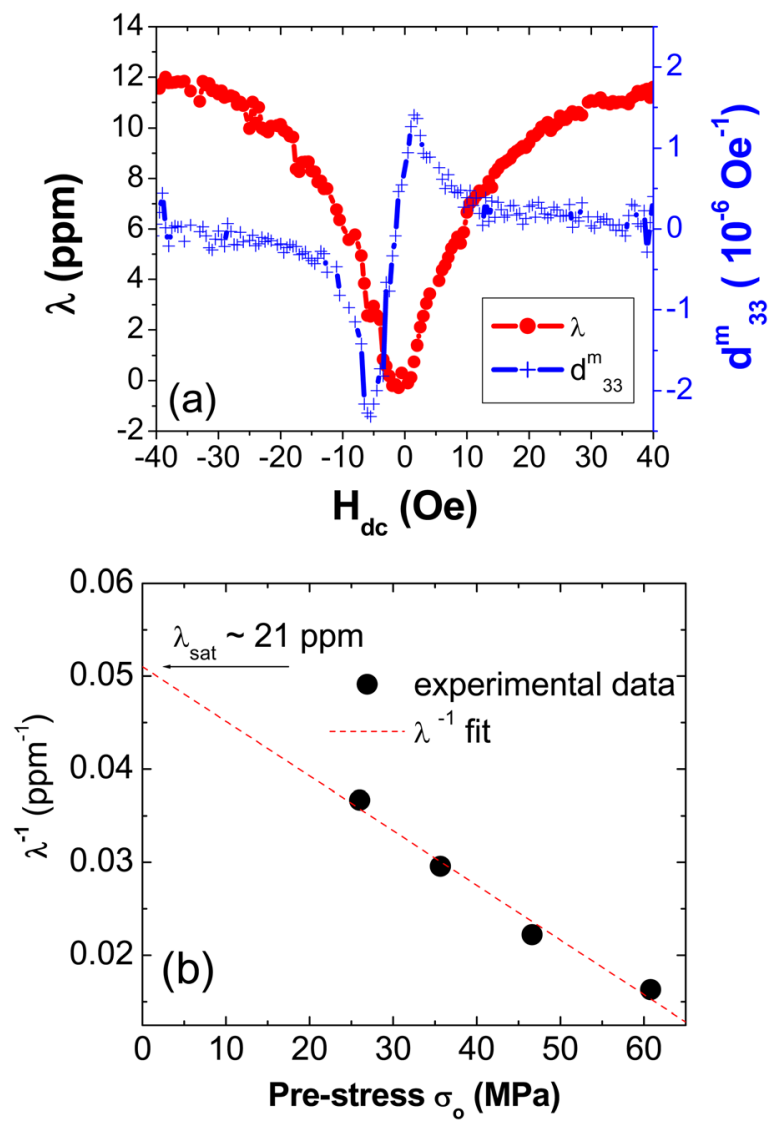

FIG. 3. (a) $\lambda$ and $d_{m}$ as a function of magnetic field bias. (b) $\lambda_{\text {sat }}$ as a function of tension.

$\lambda_{\text {sat }}$ was also measured directly with a capacitance probe (MTI Accumeasure 9000). A small piece of copper foil was adhered to the end of the Metglas ribbon with cyanoacrylate glue. With the sample clamped on one end and the copper foil held in close proximity to the capacitance probe, an $H_{d c}$ field was applied parallel to the length of the sample and the change in length was measured as a function of $H_{d c}$ field. These data were then corrected for torque effects. The change in length at $H_{\text {sat }}$ was used to calculate $\lambda_{\text {sat }}$, resulting in a value of $\lambda_{\text {sat }}=20.8 \mathrm{ppm}$, which is in good agreement with the value found above. It should be noted that other studies have found Metglas to have $\lambda_{\text {sat }}$ values as high as 39 ppm, ${ }^{19}$ well above the value found here. Numerous techniques have been developed to measure the magnetostriction constant $(\lambda)$ including both direct and indirect methods. ${ }^{20-23}$ However, considerable debate continues with regard to the accuracy of each method. The discrepancy in $\lambda_{\text {sat }}$ values may be due to the effects of material defects and dislocations due to handling, or internal stress caused by material deposition and sample preparation methods. These factors likely influence the measured magnetostriction, ${ }^{23}$ but are rarely accounted for in measurement techniques. Therefore, experimentally measured $\lambda$ may be considerably smaller than that measured in the virgin material.

In order to demonstrate the linear and anhysteretic behavior of this sensor concept, the resonator was placed in an $H_{d c}$ field of $7 \mathrm{Oe}$ to maximize sensitivity, and an additional AC magnetic field was applied with a frequency of $1 \mathrm{~Hz}$. The resonance frequency shift was measured at a variety of $H_{a c}$ amplitudes. FFTs were taken for both the $H_{a c}$ signal and the resonance frequency signal, and the amplitudes of the spectra at $1 \mathrm{~Hz}$ were recorded. Plotting the amplitude of the resonance frequency signal as a function of the $H_{a c}$ amplitude, as seen in Figure 4(a), shows that the amplitude of the resonance frequency decreases linearly with that of the $H_{a c}$ signal until approximately $\left|H_{a c}\right|=2 \mu \mathrm{T}$. At this point, there is no significant decrease in resonance frequency amplitude with $H_{a c}$, indicating that noise has begun to dominate the resonance frequency signal.

The sensor can be further characterized by calculating the equivalent magnetic noise floor, defined as $\left(S_{y}(f)\right)^{1 / 2}(d f / d H)^{-1}$, where $S_{y}(f)$ is the frequency power spectral density and $d f / d H$ is the sensor sensitivity. We will first calculate the sensitivity by utilizing the linearity of the frequency and magnetic field in the vicinity of maximum sensitivity, expressed as

$$
\frac{\Delta f}{f_{1}} \approx \frac{\Delta \sigma}{2 \sigma} \approx \frac{\lambda_{s a t} \Delta H}{2 H_{0}},
$$

where $H_{0}$ is the operating magnetic field bias (typically at maximum sensitivity), $\Delta f$ is the minimum resolvable frequency shift as determined by the Allan deviation, and $f_{1}$ is the resonance frequency at the operating magnetic field bias. By rearranging Eq. (4) and taking the limit of $\Delta f / \Delta H$, the sensitivity of the sensor can be estimated as $\left(\lambda_{\text {sat }} f_{1}\right) /\left(2 H_{0}\right)$. At the lowest tested tension, this corresponds to $d f / d H$ $\approx(12 \mathrm{ppm})(550 \mathrm{~Hz}) / 2\left(7 \times 10^{-4} \mathrm{~T}\right)=4.7 \mathrm{~Hz} / \mathrm{T}$.

The power spectral density of the frequency fluctuations can be computed using the Allan variance $\sigma^{2}(\tau) .{ }^{24}$ The Allan
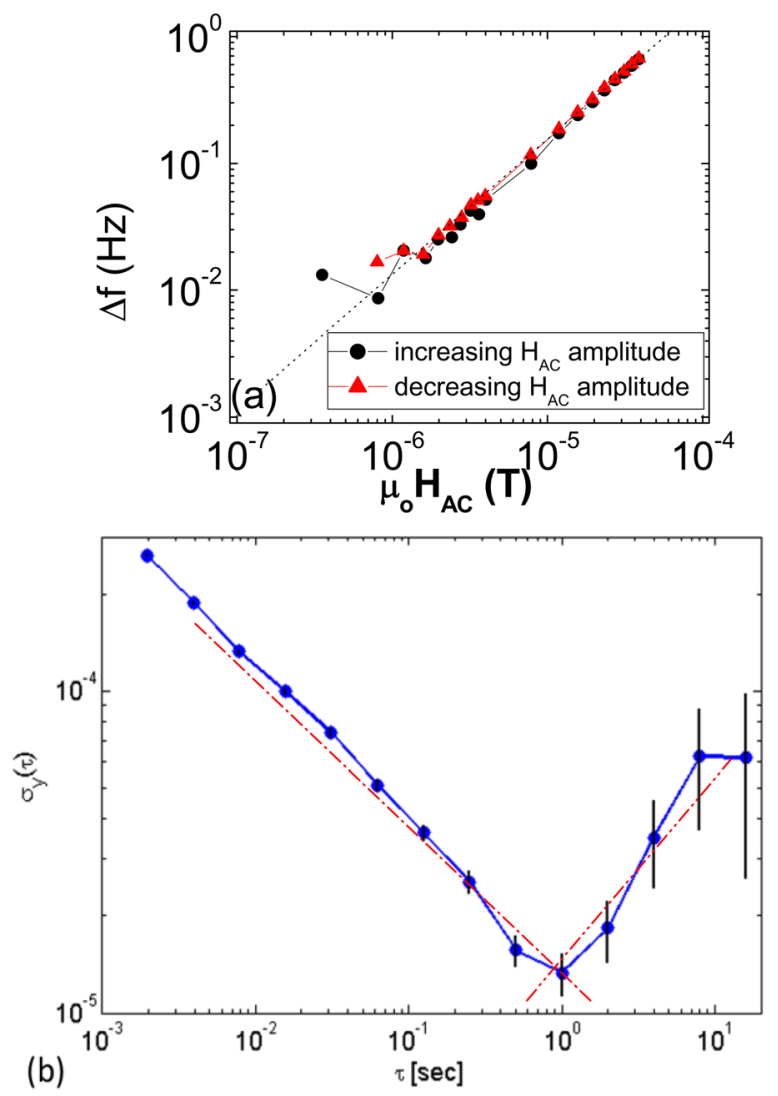

FIG. 4. (a) The linear response of $\Delta f$ vs. $H_{a c}$ and (b) Allan deviation of $\Delta f / f_{1}$. Red dashed lines show the relevant Allan deviation slopes. 
variance measures the frequency stability of the system output as a function of sampling period, $\tau$, and effectively characterizes the influence of different power-law noise types. It can be expressed as a function of the power spectral density $S(t)$ of the system output as

$$
\begin{aligned}
\sigma^{2}(\tau) & \approx \frac{1}{2(N-1)} \sum_{k=1}^{N-1}\left(\bar{S}_{k+1}-\bar{S}_{k}\right)^{2} \\
& =2 \int_{0}^{+\infty} S(f) \frac{\sin ^{4}(\pi f \tau)}{(\pi f \tau)^{2}} d f \approx \sum_{k=-2}^{2} h_{k} g(\tau),
\end{aligned}
$$

where $N$ is the sampling number and $S(f)$ is the power spectral density of $S(t)$ and $g(t)$ which are functions of $\tau$. It implies that each noise source behavior could be characterized by a different slope on the Allan variance plot. The plot shown in Figure 4(b) can be approximated by only two slopes, positive and negative unity (shown as red dashed lines), so we can deduce that the power spectral density of $S_{y}(f)$ is dominated by white frequency and random walk frequency power law spectral density contributions. The power coefficients of these dominant noise sources, $h_{0}$ and $h_{-2}$, respectively, can be calculated by fitting the relevant sections of the Allan variance plot, ${ }^{24}$ such that

$$
\begin{gathered}
\sigma_{y}^{2}(\tau) \approx \frac{\left(1.33 \times 10^{-5}\right)^{2}}{\tau} \approx \frac{h_{0}}{2 \tau}, \\
\sigma_{y}^{2}(\tau) \approx\left(1.33 \times 10^{-5}\right)^{2} \tau \approx \frac{(2 \pi)^{2}}{6} h_{-2} \tau .
\end{gathered}
$$

Using these power coefficients, the frequency power spectral density can be computed as

$$
S_{y}(f)=\sum_{n=-2}^{2} h_{n} f^{n} \approx h_{0}+\frac{h_{-2}}{f^{2}} \approx\left[354+\frac{27}{f^{2}}\right] \times 10^{-12}
$$

and the equivalent magnetic noise floor is estimated as

$$
b_{n}(f)=\frac{2 H_{0}}{\lambda_{\text {sat }} f_{1}} \sqrt{\sum_{n=-2}^{2} h_{n} f^{n}} \approx \frac{1}{4.7} \sqrt{\left[354+\frac{27}{f^{2}}\right]} \times 10^{-6},
$$

which yields a white noise floor of approximately $4 \mu \mathrm{T} / \sqrt{ } \mathrm{Hz}$. As can be seen from Eq. (8), the equivalent magnetic noise floor decreases as resonance frequency increases. The benefits of higher resonance frequency can be utilized in MEMStype resonators, which have recently been studied extensively. ${ }^{25-28}$

In summary, we have demonstrated the use of a simple magnetic field sensor composed of a doubly clamped Metglas resonator, and have examined the sensor performance as a function of uni-axial stress. Resonance frequency was tracked in real time as a function of magnetic field bias using a feedback loop based on the quadrature of the excited motion. In both the mathematical model and experimental results, it was seen that increasing tension results in lower $\lambda_{\text {sat }}$ values and, in turn, lower $\Delta f$ values. Furthermore, the sensitivity was found to be $4.7 \mathrm{~Hz} / \mathrm{T}$ at a DC bias of $700 \mu \mathrm{T}$ and the resolution was calculated to be approximately $4 \mu \mathrm{T}$ at $1 \mathrm{~Hz}$. This stress controllable Metglas resonator may ultimately be used to design a complete ME sensor, in which Metglas is paired with a piezoelectric material, such as PZT fibers ${ }^{11,12}$ or a ferroelectric thin film..$^{26-28}$

The authors would like to acknowledge the Office of Naval Research and the In-house Laboratory Independent Research (ILIR) Program at the Naval Undersea Warfare Center (NUWC).

${ }^{1}$ C.-W. Nan, M. I. Bichurin, S. Dong. D. Viehland, and G. Srinivasan, J. Appl. Phys. 103, 031101 (2008).

${ }^{2}$ G. Srinivasan, Annu. Rev. Mater. Res. 40, 153 (2010).

${ }^{3}$ J. Y. Zhai, Z. P. Xing, S. X. Dong, J. F. Li, and D. Viehland, J. Am. Ceram. Soc. 91, 351-358 (2008).

${ }^{4}$ S. X. Dong, J.-F. Li, and D. Viehland, Appl. Phys. Lett. 85, 2307 (2004).

${ }^{5}$ J. Y. Zhai, S. X. Dong, J.-F. Li, D. Viehland, and M. I. Bichurin, J. Appl. Phys. 100, 124509 (2006).

${ }^{6}$ S. X. Dong, J. F. Li, and D. Viehland, Appl. Phys. Lett. 83, 2265 (2003).

${ }^{7}$ S. X. Dong, J. Y. Zhai, N. G. Wang, F. M. Bai, J.-F. Li, D. Viehland, and T. A. Lograsso, Appl. Phys. Lett. 87, 222504 (2005).

${ }^{8}$ K. Mori and M. Wuttig, Appl. Phys. Lett. 81, 100 (2002).

${ }^{9}$ J. Y. Zhai, S. X. Dong, Z. P. Xing, J.-F. Li, and D. Viehland, Appl. Phys. Lett. 89, 083507 (2006).

${ }^{10}$ J. Y. Zhai, S. X. Dong, Z. P. Xing, J.-F. Li, and D. Viehland, Appl. Phys. Lett. 88, 062510 (2006).

${ }^{11}$ S. X. Dong, J. Y. Zhai, J.-F. Li, and D. Viehland, Appl. Phys. Lett. 89, 252904 (2006).

${ }^{12}$ M. H. Li, D. Berry, J. Das, D. Gray, J. F. Li, and D. Viehland, J. Am. Ceram. Soc. 94, 3738-3741 (2011).

${ }^{13}$ M.-H. Li, J.-Q. Gao, Y.-J. Wang, D. Gray, J.-F. Li, and D. Viehland, J. Appl. Phys. 111, 104504 (2012).

${ }^{14}$ J. R. Petrie, J. Fine, S. Mandal, G. Sreenvasulu, G. Srinivasan, and A. S. Edelstein, Appl. Phys. Lett. 99, 043504 (2011).

${ }^{15}$ S. X. Dong, J.-F. Li, and D. Viehland, Appl. Phys. Lett. 83, 4812 (2003).

${ }^{16}$ S. C. Masmanidis, H. X. Tang, E. B. Myers, M. Li, K. De Greve, G. Vermeulen, W. Van Roy, and M. L. Roukes, Phys. Rev. Lett. 95, 187206 (2005).

${ }^{17}$ P. Finkel, S. Lofland, and E. Garrity, Appl. Phys. Lett. 94, 072502 (2009).

${ }^{18}$ P. Finkel, J. Bonini, E. Garrity, K. Bussman, J. Gao, J. F. Li, S. E. Lofland, and D. Viehland, Appl. Phys. Lett. 98, 092905 (2011).

${ }^{19}$ Y. J. Wang, D. Gray, D. Berry, J. Q. Gao, M. H. Li, J. F. Li, and D. Viehland, Adv. Mater. 23, 4111 (2011).

${ }^{20}$ E. T. Lacheisserie and J. C. Peuzin, J. Magn. Magn. Mater. 136, 189-196 (1994).

${ }^{21}$ M. L. Spano, K. B. Hathaway, and H. T. Savage, J. Appl. Phys. 53, 2667-2669 (1982).

${ }^{22}$ K. Narita, J. Yamasaki, and H. Fukunaga, IEEE Trans. Magn. 16, 435-439 (1980).

${ }^{23}$ P. T. Squire, Meas. Sci. Technol. 5, 67-81 (1994).

${ }^{24}$ D. W. Allan, J. H. Shoaf, and D. Halford, NBS Monogr. 140, 151-204 (1974).

${ }^{25}$ B. Gojdka, R. Jahns, K. Meurisch, H. Greve, R. Adelung, E. Quandt, R. Knöchel, and F. Faupel, Appl. Phys. Lett. 99, 223502 (2011).

${ }^{26}$ J. H. Lee, T. S. Kim, and K. H. Yoon, Appl. Phys. Lett. 84, 3187 (2004).

${ }^{27}$ P. Zhao, Z. Zhao, D. Hunter, R. Suchoski, C. Gao, S. Mathews, M. Wuttig, and I. Takeuchi, Appl. Phys. Lett. 94, 243507 (2009).

${ }^{28}$ H. Greve, E. Woltermann, H.-J. Quenzer, B. Wagner, and E. Quandt, Appl. Phys. Lett. 96, 182501 (2010). 